\title{
Testing Asteroseismic Scaling Relations with Interferometry
}

T. R. White ${ }^{1, a}$, V. Silva Aguirre ${ }^{2}$, T. Boyajian ${ }^{3}$, O. Creevey ${ }^{4}$, D. Huber ${ }^{5,6,7}$, K. von Braun ${ }^{8}$,

T. R. Bedding ${ }^{7,2}$, Y. Elsworth ${ }^{8,2}$, S. Hekker ${ }^{10,2}$, D. Stello ${ }^{7,2}$, and A. Weiss ${ }^{11}$

1 Institut für Astrophysik, Georg-August-Universität Göttingen, Friedrich-Hund-Platz 1, 37077 Göttingen, Germany

2 Stellar Astrophysics Centre, Department of Physics and Astronomy, Aarhus University, Ny Munkegade 120, DK-8000 Aarhus C, Denmark

3 Department of Astronomy, Yale University, 260 Whitney Ave, New Haven, CT 06520, USA

4 Institut d'Astrophysique Spatiale, Université Paris XI - CNRS (UMR8617), Batiment 121, 91405 Orsay Cedex, France

5 NASA Ames Research Centre, Moffett Field, CA 94035, USA

6 SETI Institute, 189 Bernardo Avenue, Mountain View, CA 94043, USA

7 Sydney Institute for Astronomy (SIfA), School of Physics, University of Sydney, NSW 2006, Australia

8 Max-Planck-Institute for Astronomy (MPIA), Königstuhl 17, 69117 Heidelberg, Germany

9 School of Physics and Astronomy, University of Birmingham, Birmingham B15 2TT, UK

10 Max-Planck-Institut für Sonnensystemforschung, Justus-von-Liebig-Weg 3, 37077 Göttingen, Germany

11 Max-Planck-Institut für Astrophysik, Karl-Schwarzschild-Str. 1, 85741 Garching bei München, Germany

\begin{abstract}
The asteroseismic scaling relations for the frequency of maximum oscillation power, $v_{\max }$, and the large frequency separation, $\Delta v$, provide an easy way to directly determine the masses and radii of stars with detected solar-like oscillations. With the vast amount of data available from the CoRoT and Kepler missions, the convenience of the scaling relations has resulted in their wide-spread use. But how valid are the scaling relations when applied to red giants, which have a substantially different structure than the Sun? Verifying the scaling relations empirically requires independent measurements. We report on the current state and future prospects of interferometric tests of the scaling relations.
\end{abstract}

In order to fully exploit the potential of red giants as the new benchmark for Galactic studies, it is of crucial importance that the asteroseismic determination of their physical parameters results in robust and accurate values. The most straightforward way of obtaining masses, radii, and ages of red giant stars is by the asteroseismic scaling relations. These are based on extrapolations from our knowledge of the solar properties and two oscillation quantities readily extractable from the power spectrum [1-3]:

$$
\Delta v \approx \frac{\left(M / \mathrm{M}_{\odot}\right)^{(1 / 2)}}{\left(R / \mathrm{R}_{\odot}\right)^{(3 / 2)}} \Delta v_{\odot}, \quad v_{\max } \approx \frac{\left(M / \mathrm{M}_{\odot}\right)}{\left(R / \mathrm{R}_{\odot}\right)^{2} \sqrt{T_{\mathrm{eff}} / \mathrm{T}_{\mathrm{eff} \odot}}} v_{\max \odot} .
$$

Here, $v_{\max }$ is the frequency of maximum oscillation power, $\Delta v$ the separation between consecutive peaks of the same angular degree, and the $\odot$ symbol corresponds to the accurately known properties

a e-mail: twhite@astro.physik.uni-goettingen.de

This is an Open Access article distributed under the terms of the Creative Commons Attribution License 4.0, which permits unrestricted use, distribution, and reproduction in any medium, provided the original work is properly cited. 


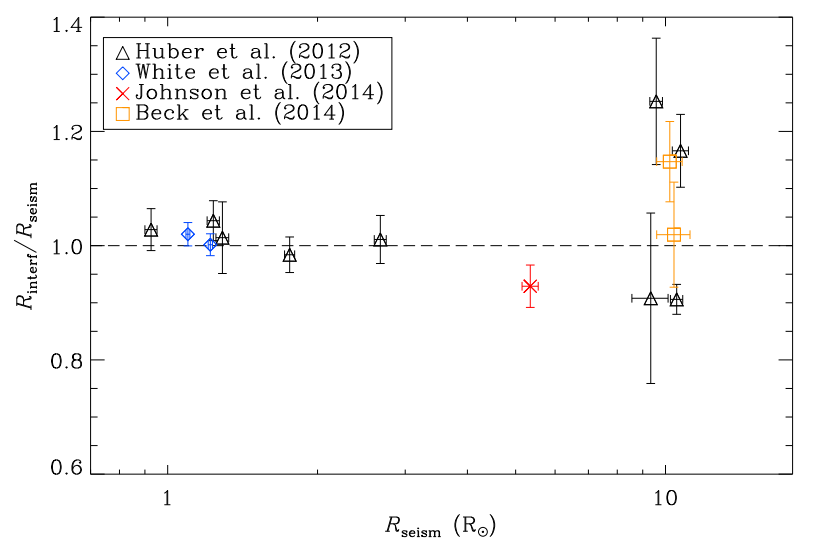

Fig. 1. Comparison of stellar radii measured using interferometry and calculated using asteroseismic scaling relations. Black triangles show stars measured by [7], the blue diamonds are $16 \mathrm{Cyg} \mathrm{A}$ and $\mathrm{B}$ [8], the red cross is HD 185351 [9], and orange squares indicate $\gamma$ Psc and $\theta^{1}$ Tau [10].

of the Sun. With a measurement of the effective temperature, $T_{\text {eff }}$, the above equations allow us to determine the stellar mass $M$ and radius $R$ [4]. Coupled to a determination of metallicity and stellar evolutionary models, the scaling relations can provide ages for red-giant stars through grid-based modelling.

Although the scaling relations do have some theoretical justification [5], they require validation across different evolutionary stages. In the case of dwarfs, tests of the asteroseismic radii have been made by comparing the predicted distances with those from Hipparcos parallaxes [6], as well as for a handful of cases where interferometric observations were feasible [7].

Figure 1 shows the comparison between asteroseismic and interferometric radii from various sources [7-10]. It can be seen that the agreement is good for dwarfs and subgiants $\left(R\right.$ below $\left.\sim 3 \mathrm{R}_{\odot}\right)$, while much larger scatter can be observed for red giant stars. These results cast some doubts over the applicability of the scaling relations as written above to all stars regardless of their evolutionary phase.

The only way forward to ensure our predictions from asteroseismic scaling relations are robust is to test and calibrate them using a sample of independently determined stellar parameters covering a large range of effective temperatures and metallicites. This requires red giant targets for which both excellent seismic and interferometric, spectroscopic, and astrometric data are available. This has not been possible with Kepler observations because the brightest giants observed have $V \sim 7$ mag and are too faint for complementary observations of sufficient precision. The precise seismic K2 observations and high-quality ground-based data of bright nearby giants will allow us to subject the scaling relations to the most demanding tests.

\section{References}

1. Ulrich, R. K., ApJ, 306, (1986) L37

2. Brown, T. M., Gilliland, R. L., Noyes, R. W., \& Ramsey, L. W. ApJ 1991, (1991) 599

3. Kjeldsen, H., \& Bedding, T. R. A\&A 293, (1995) 87

4. Stello, D., Bruntt, H., Preston, H., \& Buzasi, D. ApJ 647, (2008) L53

5. Belkacem, K., Goupil, M. J., Dupret, M. A., et al. A\&A 530, (2011), A142

6. Silva Aguirre, V., Casagrande, L., Basu, S., et al. ApJ 757, (2012) 99

7. Huber, D., Ireland, M. J., Bedding, T. R., et al. ApJ 760, (2012) 32

8. White, T. R., Huber, D., Maestro, V., et al. 2013, MNRAS 433, (2013) 1262

9. Johnson, J. A., Huber, D., Boyajian, T., et al. ApJ 794, (2014) 15

10. Beck, P. G., Kambe, E., Hillen, M., et al. A\&A, (2014) in press 